\title{
Surgical Instruction Generation with Transformers
}

\author{
Jinglu Zhang ${ }^{1}$, Yinyu $\mathrm{Nie}^{2, *}$, Jian Chang ${ }^{1}$, and Jian Jun Zhang ${ }^{1}$ \\ 1 National Centre for Computer Animation (NCCA), Bournemouth University, UK \\ 2 Technical University of Munich
}

\begin{abstract}
Automatic surgical instruction generation is a prerequisite towards intra-operative context-aware surgical assistance. However, generating instructions from surgical scenes is challenging, as it requires jointly understanding the surgical activity of current view and modelling relationships between visual information and textual description. Inspired by the neural machine translation and imaging captioning tasks in open domain, we introduce a transformer-backboned encoder-decoder network with self-critical reinforcement learning to generate instructions from surgical images. We evaluate the effectiveness of our method on DAISI dataset, which includes 290 procedures from various medical disciplines. Our approach outperforms the existing baseline over all caption evaluation metrics. The results demonstrate the benefits of the encoderdecoder structure backboned by transformer in handling multimodal context.
\end{abstract}

Keywords: surgical instruction generation - transformer · image captioning $\cdot$ reinforcement learning

\section{Introduction}

Surgical instruction generation is a task of automatically generating a natural language sentence to guide surgeons of how to perform the operation based on the current surgical view. It is an essential component towards building contextaware surgical system, which aims to utilize available information inside the operation room to provide clinicians with contextual support at appropriate time. Moreover, when on-site mentoring is unavailable or a rare case is detected, providing intra-operative surgical instructions by expert surgeons is imperative. However, surgical data has high heterogeneity even for the same type of surgery due to different surgical skill level, medical condition, and patient specific situation. Accordingly, understanding surgical content and generating a natural language sentence to guide the procedure is challenging.

\footnotetext{
*Corresponding email: yinyu.nie@tum.de
} 
Previously, telementoring [5], which exchanges medical information through video and audio in real time, has been proved as an efficient solution for intraoperative guidance, including pointing out target anatomical structure from the monitor, controlling the camera or the robotic arm, etc. Nonetheless, telementoring is limited by the cost of specific equipment and software, the high demand of transport speed, and legal and ethic issues [5,9]. With the huge development of related techniques of context-aware surgical assistance, understanding and analyzing the surgical activities inside the operation room opens up the possibility of providing intra-operative assistance for surgeons. Most of the existing researches focus on surgical phases and fine-grained gestures recognition [22,10,27]. However, these methods can be regarded as the segmentation and classification problems based on pre-defined phases and gestures, thus have no ability of generating the unseen instructions.

The most related research topic to us is medical report generation $[12,7,6]$, which describes the impression, findings, tags, etc. of a patient in reference to the radiology or pathology. One of the earliest medical report generation works based on natural language is [12], which jointly predicts tags and generates long paragraphs with co-attention and hierarchical LSTM. More recently, [7] improves the transformer model [23] by designing a relational memory to record key information of the generation process and provides a memory-driven layer normalization for transformer decoder. Despite the challenges, medical reports also have their own discriminating characters. They often share predefined topics and follow similar writing templates, while surgical instruction generation with natural language has no template to follow.

To our best knowledge, [20] is the only prior work for surgical instruction generation. In their work, the authors create the Database for AI Surgical Instruction dataset (DAISI) and use a bidirectional recurrent neural network (RNN) to generate the description for a surgical image. However their work has two limitations. For one, although RNNs are designed for sequence generation with arbitrary length, they suffer from the essential vanishing gradient problem [17]. For another, they apply the BLEU [16] score as the only evaluation metric, which is insufficient for natural language evaluation.

In this paper, inspired by the great performance of transformer model in machine translation [23] and image captioning [8] from the open domain, we build our network with an encoder-decoder fully backboned transformer to generate surgical instructions. Taking an surgical image as the input, we first extract its visual attention features by a fine-tuned ResNet-101 module. Then the encoder attention blocks, decoder attention blocks, and encoder-decoder attention blocks model the dependencies for visual features, textual features, and visualtextural relational features, respectively. On the other hand, sequence generation models are often trained using the cross-entropy (XE) loss and evaluated using non-differential metrics such as BLEU, CIDEr [24], etc. In order to alleviate the mismatch between training and testing and improve the evaluation performance, we apply the reinforcement learning based self-critical approach [19] to directly optimize the CIDEr score. Experimentally, we extensively explore 
the performance of different baselines (LSTM-based fully connected and softattention models) on DAISI dataset [20]. The experiments demonstrate that our transformer-backboned architecture outperforms the existing methods as well as our other proposed baselines. The promising instructions generated from the network bring potential value in clinical practice.

\section{Methodology}

In this section, we introduce our framework in details. It involves two submodules: 1) the transformer-backboned encoder-decoder structure for surgical instruction generation (see section 2.1);2) the self-critical reinforcement learning for optimizing the CIDEr score (see section 2.2).

\subsection{Encoder-Decoder with Transformer Backbone}

The whole encoder-decoder structure can be seen in Figure 1. Following the modern learning paradigm, we design this kind of structure to encode latent features from images and decode them into natural languages. Before our network, a ResNet-101 [11] is adopted to output $14 \times 14 \times 2048$ image features, which are afterwards embeded by a linear embedding layer to reduce the dimension to $14 \times 14 \times 512$ followed by a ReLU and a dropout layer. Subsequently, our encoder firstly processes the flattened spatial features $(196 \times 512)$ and produces non-local relationships between image regions. Then the decoder takes hidden attentive representation from the encoder outputs and generates the corresponding instruction with natural language. The essential attention mechanism behind the transformer is called scaled dot-product attention [23], which is defined as:

$$
\operatorname{Attention}(Q, K, V)=\operatorname{Softmax}\left(\frac{Q K^{T}}{\sqrt{d}}\right) V
$$

where $Q$ is the packed query matrix, $K$ and $V$ are packed key-value pairs, and $d$ is a scaling factor (equals to the dimension of $K$ ). It calculates a weighted sum of the values based on the similarity distribution between the query with all the keys.

The whole encoder is a stack of 6 attention blocks with identical structure. Specifically, each block consists of a multi-head self-attention layer ( 8 heads) and a position-wise feed-forward network. The multi-head self-attention layer is represented as:

$$
\begin{aligned}
\operatorname{MultiHead}(Q, K, V) & =\text { Concat }\left(\text { head }_{1}, \ldots, \text { head }_{h}\right) W^{O} \\
\text { head }_{\mathrm{i}} & =\operatorname{Attention}\left(Q W_{i}^{Q}, K W_{i}^{K}, V W_{i}^{V}\right)
\end{aligned}
$$

where matrices $W_{i}^{Q}, W_{i}^{Q}, W_{i}^{Q}$ and $W^{O}$ are projection parameters to be learned during the training phase. Different linear transformations are applied to the 
queries, values, and keys for each attention head. A simple position-wise fully connected feed-forward network is then applied to each attention layer:

$$
\operatorname{FFN}(\mathrm{x})=\max \left(0, x W_{1}+b_{1}\right) W_{2}+b_{2},
$$

where $W_{1}, W_{2}$ and $b_{1}, b_{2}$ are corresponding weights and biases for two fully connected layers.

The input of the decoder is the hidden representation exported from the last encoder layer. The decoder also consists of six identical blocks, where each has two multi-head attention layers (a decoder self-attention layer and an encoderdecoder attention layer) and one fully connected feed-forward network. Every decoder self-attention layer is masked to prevent from attending to future locations. For further explanation of the decoder, please refer to the original transformer paper.[23].

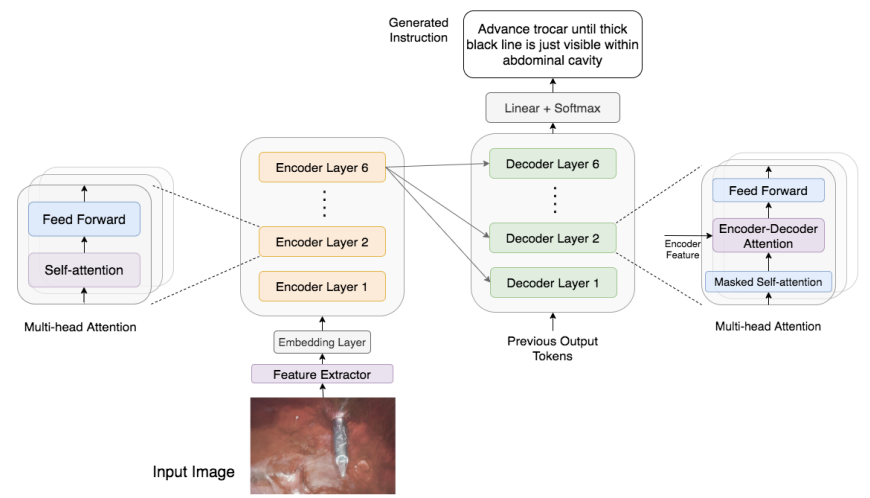

Fig. 1. The transformer-backboned surgical instruction generation architecture

\subsection{Reinforcement Learning}

Sequence generation models are often trained in "Teacher-Forcing" [4] mode, which inputs the ground-truth to maximize the likelihood of next prediction during training and uses previously generated words from the model distribution to predict the next word during test time. In order to bridge this gap, we apply the self-critical reinforcement learning as proposed in [19]. After pre-training the model with standard word-level XE loss, the CIDEr score [24] is directly optimized as the reward. All the detailed formula derivation can be found in [19].

\section{Evaluation}

\subsection{Experimental Settings}

Dataset Description. We evaluate our approach on DAISI dataset [20], which contains 17,255 color images from 290 medical procedures, including external 
fetal monitoring, laparoscopic sleeve gastrectomy, laparoscopic ventral hernia repair, etc. The availability of the dataset is upon request ${ }^{3}$. Every procedure consists of few images with their corresponding instruction texts. We further clean the dataset by deleting noisy and irrelevant images and descriptions. Finally, there are 16,413 images in total with one caption for each image. While some surgical procedures have only one sample due to the limited dataset size, we split the data in per image manner. We assign 13,094 images for training, 1,646 for validation, and 1,673 for testing.

Text Preprocessing. Text preprocessing is a significant step to transform the text into a more analyzable and predictable format for the deep learning model. Raw text instructions need to be preprocessed to learn meaningful features and not overfit on irrelevant noise. We follow these steps to clean the text instruction: 1) Converting all words to lower case; 2) Expanding abbreviations, including medical abbreviations (e.g. 'a.' to 'artery') and English contractions (e.g. i've to 'i have'); 3) Removing numbers, punctuation, and whitespace; 4) Tokenizing the sentence into words.

We further set the threshold of the sentence length to 16, label any word count less than five as 'UNK', and build a vocabulary of size 2212 words.

Evaluation Metrics. Besides the instruction generation task, how to automatically evaluate the generated sentences has become increasingly important. The key idea is to measure the correlation of generated captions with human judgments. Following most of the image captioning methods, we apply 1-4 gram BLEU [16], Rouge-L [14], METEOR [3], CIDEr [24], and SPICE [1] to evaluate our model, while the first three metrics are originated from machine translation and the last two metrics are specifically designed for image captioning.

\subsection{Implementation and Training Details}

All the models are implemented in PyTorch and trained on a single NVIDIA GeForce GTX 1080 graphics card. We first train our model with a word-level cross-entropy (XE) loss, then optimize the model using reinforcement learning. It takes around 30 hours for the training process (30 epochs for general XE loss, and 30 epochs for reinforcement learning). During the XE training process, the model is trained to predict the next word given previous ground-truth word, while the reinforcement learning process is trained to predict next word based on the previous prediction. It takes around 30 hours for the training process (30 epochs for general XE loss, and 30 epochs for reinforcement learning)

Transformer Encoder-Decoder. We use ResNet-101 [11] pre-trained on ImageNet classification task to extract image features. A spatially adaptive maxpooling layer is applied after the final convolution layer. It ends up with a fixed size of $14 \times 14 \times 2048$-d (196 image regions in total) output. For the XE training, we initialize the learning rate to $3 \times 10^{-4}$ and follow the learning rate scheduling strategy with 20000 warm-up steps for 30 epochs. During the self-critical

\footnotetext{
${ }^{3}$ https://engineering.purdue.edu/starproj/
} 
evaluation, we use a fixed learning rate of $1 \times 10^{-5}$ for another 30 epochs. Both models are optimized using ADAM optimizer [13] with a batch size of 5 .

LSTM-based Models. In order to comprehensively evaluate the surgical instruction generation task, we implement two additional models for comparison and discussion, namely LSTM model and LSTM-based soft-attention model similarly to $[26,25]$. For LSTM model, images are encoded to 2048 dimension vectors with the final convolution layer of ResNet-101 followed by an average pooling layer. The LSTM-based soft-attention model shares the same image feature maps with transformer model. For both models, the image embedding, words embedding dimension and LSTM hidden state size are set to 512 .

\section{Results and Discussion}

\subsection{Comparison with the State-of-the-Art}

We clean the original dataset [20] by removing noisy and wrong image-text pairs. Thus a new benchmark is required. As the code in [20] is not publicly available, we re-implement their Bi-RNN model. The 4096 dimensional image features are extracted using the last convolutional layer from a pre-trained VGG16 [21]. The $\mathrm{Bi}-\mathrm{RNN}$ model is trained with 50 epochs by the initial learning rate at $5 \times 10^{-4}$ and the batch size at 10 . Table 1 compares our proposed models with [20], which shows that Bi-RNN has relatively lower performance, especially for the 3-gram and 4-gram BLEU scores (11.3\% and 9.3\%) compared with ours (46.4\% and $44.9 \%)$. In BLEU score evaluation, long $n$ - gram score measures the fluency of the instruction. It can be concluded that Bi-RNN is not capable of generating "human-like" instructions.

LSTM model achieves slightly better performance than LSTM-based softattention approach, and the transformer model outperforms all the others in all metrics. This indicates that the conventional RNN-based methods have limited ability of catching the dependencies between image features and text information. While transformer-backboned encoder-decoder layers can encode the dependencies for image pixels, the self-attention layers in decoder are able to model dependencies for textual information, and the encoder-decoder attention

builds the relationship between image and textual features. Figure 2 shows some visualization samples using the proposed transformer-backboned framework.

\subsection{Effects of Reinforcement Learning}

To further explore the functionality of each design in our network, we decouple three networks and design an ablative experiment in six settings: (1) LSTM only; (2) LSTM + reinforcement learning; (3) LSTM + soft-attention; (4) LSTM + soft-attention + reinforcement learning; (5) Transformer only; (6) Transformer + reinforcement learning. The experiment results are shown in Table 2.

(1) v.s. (3): We add the soft-attention module on the top of LSTM to sequentially attend to different parts of image and aggregate information, but it 
Table 1. Comparison with the state-of-the-art [20] for surgical instruction generation task. B1, B2, B3, B4, C, M, R and S stands for 1-4 gram BLEU, CIDEr, METEOR, ROUGE-L and SPICE score respectively.

\begin{tabular}{lcccccccc}
\hline $\begin{array}{l}\text { Surgical } \\
\text { Instruction }\end{array}$ & $B 1$ & $B 2$ & $B 3$ & $B 4$ & $C$ & $M$ & $R$ & $S$ \\
\hline DAISI (Bi-RNN) & 21.0 & 14.4 & 11.3 & 9.3 & 8.32 & 10.3 & 22.0 & 12.1 \\
LSTM & 43.7 & 39.4 & 37.3 & 36.2 & 34.0 & 24.9 & 44.6 & 40.2 \\
LSTM + soft-attn & 43.2 & 38.7 & 36.3 & 34.9 & 32.4 & 24.3 & 43.7 & 38.0 \\
Transformer + rl & $\mathbf{5 2 . 8}$ & $\mathbf{4 8 . 7}$ & $\mathbf{4 6 . 4}$ & $\mathbf{4 4 . 9}$ & $\mathbf{4 2 . 7}$ & $\mathbf{3 0 . 7}$ & $\mathbf{5 3 . 1}$ & $\mathbf{4 8 . 4}$ \\
\hline
\end{tabular}

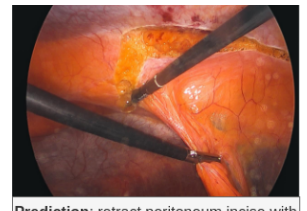

Prediction: retract peritoneum incise w scissors and electrocaute GT: while retracting peritoneum, identify correct plane by thin areolar tissue

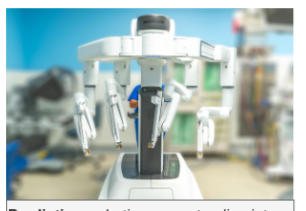
Prediction: robotic arms extending into first stage of proper draping position GT: robotic arms extending into first stage of proper draping position

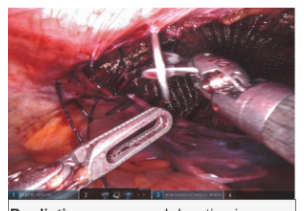

Prediction: once mesh location is secured with suture, close the
flap with running vlock suture

GT: once mesh location is secured wi suture, close the peritoneal flap with suture, close the peri
running vlock suture
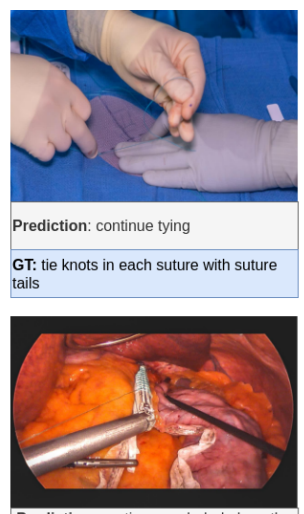

Prediction: continue cephalad along the care to avoid narrowing the stomach GT: continue cephalad along the greater curvature of the stomach, taking care to avoid narrowing the stomach and spiraling the staple line

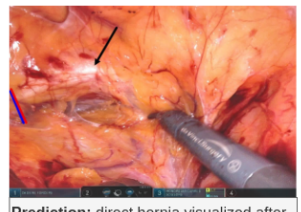

Prediction: direct hernia visualized after flap UNK, red and blue lines equals
inferior epigastric vessels UNK box GT: complete adequate inferior dissectio to below coopers ligament to allow for
appropriate mesh overlay
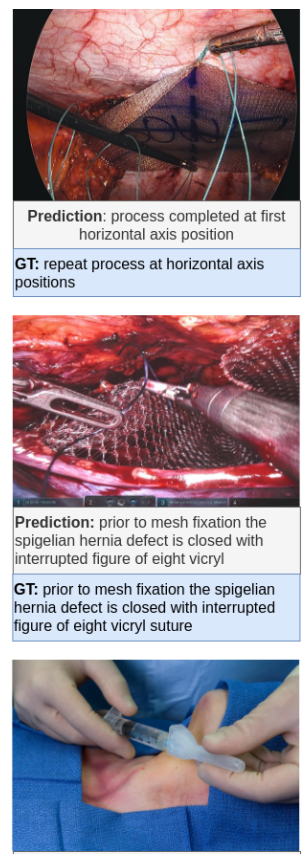

Prediction: connect syringe to large gauge needle GT: remove needle ca

Fig. 2. Qualitative results with transformer-backboned encoder-decoder framework.

performs slightly worse (around 1\% for each evaluation standard) than the baseline model. This indicates that simple soft-attention mechanism cannot build the correlation between salient pixels and the next word prediction.

(1) v.s. (3) v.s. (5): Without using any recurrent neural units as LSTMbased models, transformer-backboned model only use the attention mechanism to encode the image information and decode its corresponding text instruction. Transformer-backboned model achieves better performance than two LSTM models, which demonstrate its ability in handling multi-modal contexts. 
(1) v.s. (2), (3) v.s. (4), and (5) v.s. (6): During the training procedure, we first train each model with standard XE loss, then we add the reinforcement learning block to optimize the CIDEr score directly. From the results, it can be seen that not only the CIDEr score, but also the performance of other evaluation metrics has been lifted. Specifically, we observe a significant increasing when using reinforcement training after the transformer-backboned model.

Table 2. Ablative study to explore the influence of reinforcement learning. B1, B2, B3, B4, C, M, R and S stands for 1-4 gram BLEU, CIDEr, METEOR, ROUGE-L and SPICE score respectively.

\begin{tabular}{lcccccccc}
\hline $\begin{array}{l}\text { Surgical } \\
\text { Instruction }\end{array}$ & $B 1$ & $B 2$ & $B 3$ & $B 4$ & $C$ & $M$ & $R$ & $S$ \\
\hline LSTM & 43.7 & 39.4 & 37.3 & 36.2 & 34.0 & 24.9 & 44.6 & 40.2 \\
LSTM + rl & 44.6 & 40.3 & 38.3 & 37.1 & 35.1 & 25.4 & 45.3 & 41.1 \\
LSTM + attn & 43.2 & 38.7 & 36.3 & 34.9 & 32.4 & 24.3 & 43.7 & 38.0 \\
LSTM + attn + rl & 43.4 & 38.8 & 36.4 & 34.8 & 33.1 & 24.8 & 44.1 & 38.5 \\
Transformer & 45.5 & 41 & 38.7 & 37.2 & 34 & 25.6 & 44.3 & 39.7 \\
Transformer + rl & $\mathbf{5 2 . 8}$ & $\mathbf{4 8 . 7}$ & $\mathbf{4 6 . 4}$ & $\mathbf{4 4 . 9}$ & $\mathbf{4 2 . 7}$ & $\mathbf{3 0 . 7}$ & $\mathbf{5 3 . 1}$ & $\mathbf{4 8 . 4}$ \\
\hline
\end{tabular}

\subsection{Limitations and Challenges}

In this part, we discuss the current challenges and limitations for automatic surgical instruction generation.

1. Small dataset size. Deep learning algorithms often require huge amount of data to tune the parameters and prevent overfitting, e.g., COCO dataset [15] has more than $120 \mathrm{~K}$ samples. Surgical instruction generation is a multimodal problem, which relates visual, text, and the relationship between them. Therefore, the solution space is much larger than other tasks (e.g. classification and segmentation). However excluding the noisy and irrelevant images, DAISI dataset only contains 16,413 images.

2. No fine-grained supervisions. In feature extraction, some image captioning algorithms use Faster R-CNN algorithm [18] to detect object bounding boxes and identify attribute features with Visual-Genome data [2]. However, obtaining semantic and attributive annotations in medical science is quite challenging since it requires expert annotators.

3. One caption per image. In real life, an image can be described in different ways. For example, COCO captioning task has equipped with 5 different reference translations for each image. Nonetheless, we have only one annotation per image. It is possible that the evaluation metrics grade an adequate caption a low score only because it does not fit the ground truth. 


\section{Conclusion}

In this paper, we propose an encoder-decoder architecture fully backboned by transformer to generate surgical instructions from various medical disciplines. The experiment results demonstrate that transformer architecture is capable of creating the pixel-wise patterns from self-attention encoder, developing text relationships for masked self-attention decoder, and devising the image-text dependencies from encoder-decoder attention. In order to solve the mismatching between the training and testing procedure, we optimize the model with selfcritical reinforcement learning, which takes the CIDEr score as the reward after the general cross-entropy training.

Understanding surgical activity and generating instruction is still at its early stage. Future works include collecting the large training dataset, building the specialized pre-trained model for medical images, regularizing and annotating more reference captions for surgical images.

\section{References}

1. Anderson, P., Fernando, B., Johnson, M., Gould, S.: Spice: Semantic propositional image caption evaluation. In: European Conference on Computer Vision. pp. 382398. Springer (2016)

2. Anderson, P., He, X., Buehler, C., Teney, D., Johnson, M., Gould, S., Zhang, L.: Bottom-up and top-down attention for image captioning and visual question answering. In: Proceedings of the IEEE conference on computer vision and pattern recognition. pp. 6077-6086 (2018)

3. Banerjee, S., Lavie, A.: Meteor: An automatic metric for mt evaluation with improved correlation with human judgments. In: Proceedings of the acl workshop on intrinsic and extrinsic evaluation measures for machine translation and/or summarization. pp. 65-72 (2005)

4. Bengio, S., Vinyals, O., Jaitly, N., Shazeer, N.: Scheduled sampling for sequence prediction with recurrent neural networks. In: Advances in Neural Information Processing Systems. pp. 1171-1179 (2015)

5. Bilgic, E., Turkdogan, S., Watanabe, Y., Madani, A., Landry, T., Lavigne, D., Feldman, L.S., Vassiliou, M.C.: Effectiveness of telementoring in surgery compared with on-site mentoring: a systematic review. Surgical innovation 24(4), 379-385 (2017)

6. Bustos, A., Pertusa, A., Salinas, J.M., de la Iglesia-Vayá, M.: Padchest: A large chest x-ray image dataset with multi-label annotated reports. Medical image analysis 66, 101797 (2020)

7. Chen, Z., Song, Y., Chang, T.H., Wan, X.: Generating radiology reports via memory-driven transformer. arXiv preprint arXiv:2010.16056 (2020)

8. Cornia, M., Stefanini, M., Baraldi, L., Cucchiara, R.: Meshed-memory transformer for image captioning. In: Proceedings of the IEEE/CVF Conference on Computer Vision and Pattern Recognition. pp. 10578-10587 (2020)

9. Erridge, S., Yeung, D.K., Patel, H.R., Purkayastha, S.: Telementoring of surgeons: a systematic review. Surgical innovation 26(1), 95-111 (2019) 
10. Funke, I., Bodenstedt, S., Oehme, F., von Bechtolsheim, F., Weitz, J., Speidel, S.: Using $3 \mathrm{~d}$ convolutional neural networks to learn spatiotemporal features for automatic surgical gesture recognition in video. In: International Conference on Medical Image Computing and Computer-Assisted Intervention. pp. 467-475. Springer (2019)

11. He, K., Zhang, X., Ren, S., Sun, J.: Deep residual learning for image recognition. In: Proceedings of the IEEE conference on computer vision and pattern recognition. pp. $770-778$ (2016)

12. Jing, B., Xie, P., Xing, E.: On the automatic generation of medical imaging reports. arXiv preprint arXiv:1711.08195 (2017)

13. Kingma, D.P., Ba, J.: Adam: A method for stochastic optimization. arXiv preprint arXiv:1412.6980 (2014)

14. Lin, C.Y.: Rouge: A package for automatic evaluation of summaries. In: Text summarization branches out. pp. 74-81 (2004)

15. Lin, T.Y., Maire, M., Belongie, S., Hays, J., Perona, P., Ramanan, D., Dollár, P., Zitnick, C.L.: Microsoft coco: Common objects in context. In: European conference on computer vision. pp. 740-755. Springer (2014)

16. Papineni, K., Roukos, S., Ward, T., Zhu, W.J.: Bleu: a method for automatic evaluation of machine translation. In: Proceedings of the 40th annual meeting of the Association for Computational Linguistics. pp. 311-318 (2002)

17. Pascanu, R., Mikolov, T., Bengio, Y.: On the difficulty of training recurrent neural networks. In: International conference on machine learning. pp. 1310-1318 (2013)

18. Ren, S., He, K., Girshick, R., Sun, J.: Faster r-cnn: Towards real-time object detection with region proposal networks. IEEE transactions on pattern analysis and machine intelligence 39(6), 1137-1149 (2016)

19. Rennie, S.J., Marcheret, E., Mroueh, Y., Ross, J., Goel, V.: Self-critical sequence training for image captioning. In: Proceedings of the IEEE Conference on Computer Vision and Pattern Recognition. pp. 7008-7024 (2017)

20. Rojas-Muñoz, E., Couperus, K., Wachs, J.: Daisi: Database for ai surgical instruction. arXiv preprint arXiv:2004.02809 (2020)

21. Simonyan, K., Zisserman, A.: Very deep convolutional networks for large-scale image recognition. arXiv preprint arXiv:1409.1556 (2014)

22. Twinanda, A.P., Shehata, S., Mutter, D., Marescaux, J., De Mathelin, M., Padoy, N.: Endonet: A deep architecture for recognition tasks on laparoscopic videos. IEEE transactions on medical imaging 36(1), 86-97 (2016)

23. Vaswani, A., Shazeer, N., Parmar, N., Uszkoreit, J., Jones, L., Gomez, A.N., Kaiser, Ł., Polosukhin, I.: Attention is all you need. In: Advances in neural information processing systems. pp. 5998-6008 (2017)

24. Vedantam, R., Lawrence Zitnick, C., Parikh, D.: Cider: Consensus-based image description evaluation. In: Proceedings of the IEEE conference on computer vision and pattern recognition. pp. 4566-4575 (2015)

25. Vinyals, O., Toshev, A., Bengio, S., Erhan, D.: Show and tell: A neural image caption generator. In: Proceedings of the IEEE conference on computer vision and pattern recognition. pp. 3156-3164 (2015)

26. Xu, K., Ba, J., Kiros, R., Cho, K., Courville, A., Salakhudinov, R., Zemel, R., Bengio, Y.: Show, attend and tell: Neural image caption generation with visual attention. In: International conference on machine learning. pp. 2048-2057 (2015)

27. Zhang, J., Nie, Y., Lyu, Y., Li, H., Chang, J., Yang, X., Zhang, J.J.: Symmetric dilated convolution for surgical gesture recognition. In: International Conference on Medical Image Computing and Computer-Assisted Intervention. pp. 409-418. Springer (2020) 